\title{
What is a One Dimensional Metal?
}

M. Weger, Jerusalem

(Racah Institute of Physics, Hebrew University of Jerusalem)

\section{Organic charge transfer compounds} with metallic conductivity have been the subject of intensive research for the past few years $1,2,3$ ), the most investigated metal in the class being TTF-TCNQ (Tetra thio fulvalene-tetra cyano quino dimethanide). In these materials, negatively charged molecules (acceptors) such as TCNQ are stacked in linear columns (chains), as well as the positively charged donors, such as TTF. Electrical conductivity is primarily along these columns and so these materials are known as "one dimensional metals".

Let us first ask ourselves, what is a one dimensional metal. A simple definition is, that it is a substance that possesses metallic conductivity in one direction, and does not possess metallic conductivity in the perpendicular directions. The question is, how to determine this experimentally. The conductivity tensor $\sigma$ of a onedimensional metal is rather anisotropic, $\sigma_{\|}>>\sigma_{\perp}$; however, a large anisotropy (even a few hundred) is not a sufficient condition, as metallic conductivities can vary by several orders of magnitude. It was first pointed out by Heeger that in TTF-TCNQ, the electronic mean free path $\lambda$ is several lattice constants $b$ (particularly at low temperatures) along the columns, while it is much less than the intercolumn separation perpendicular to them. Thus, the perpendicular conductivity is a diffusive, rather than a metallic, wavelike, phenomenon. More recently, the relaxation (i.e. jump) time for electronic motion perpendicular to the chains, has been determined directly, by NMR ${ }^{4}$ ). This is done by comparing the jump time between chains, $\tau_{\perp}$ to the electron spin precession frequency $\omega_{e}=\gamma_{e} H$ which is known precisely in a given field. This yields $\tau_{\perp}=10^{-11} \mathrm{~s}$ under normal conditions. The collision time for motion along the chains, $\tau_{\|}$, can be determined from the conductivity, $\sigma_{\|}=n e^{2} \tau_{\| \prime} / m^{\star}$, as well as from the electron spin diffusion constant $D_{\|}$ (also determined by NMR), $\mathrm{D}_{\|} \approx v_{F}{ }^{2} \tau_{\| \prime}$ and is $3.10^{-15} \mathrm{~s}$ under normal conditions for TCNQ ( $\sim 5.10^{-16} \mathrm{~s}$ for TTF). Thus, the electron scatters about a thousand times in a chain, before it diffuses away from it, and the one- dimensional metallic state is characterized by $\tau_{\perp}>\tau_{\|}$The transverse conductivity is given by :

$$
\begin{aligned}
& \sigma_{\perp} \approx n\left(E_{F}\right) k_{B} T \text { e } \mu ; \\
& \mu \approx e D_{\|} / k_{B} T \text { (Einstein relation) } ; \\
& D_{\|}=d^{2} / \tau_{\perp},
\end{aligned}
$$

where $d$ is the interchain distance, thus $\sigma_{\perp} \approx e^{2} n\left(E_{F}\right) d^{2 / \tau}$

A longer $\tau_{\perp}$ gives rise to a lower conductivity, in agreement with experiment.

Thus, the coherent (wavelike) motion of the electrons along the columns (of TCNQ, at least) and the diffusive (hopping) motion perpendicular to the columns, are by now firmly established, and we are by now confident that TTF-TCNQ is indeed a 1-D metal over the temperature range $53 \mathrm{~K}-300 \mathrm{~K}$. At $53 \mathrm{~K}$, TTF-TCNQ undergoes a phase-transition to a semiconducting state, while above ambient temperature, the mean free path $\lambda$ becomes so short, that $\lambda \mathrm{k}_{\mathrm{F}}<1$, and the motion is about to lose its metallic character along the columns as well.

\section{Organic Metal \\ at Helium Temperatures}

We know that a large part of our understanding of the metallic state stems from galvanomagnetic measurements conducted at helium temperatures ${ }^{5}$ ). Most measurements that determine the Fermi surface (such as magnetoresistance, de Haas Van Alpen effect, cyclotron resonance) are conducted in this temperature range. Investigation of organic metals in this temperature range should, therefore, be promising. Unfortunately, TTFTCNQ becomes semi-conducting at $T_{P}=53 \mathrm{~K}$. Application of pressure helps to extend the metallic state to lower temperatures in many materials

such as $\mathrm{V}_{2} \mathrm{O}_{3}, \mathrm{VO}_{2}$, etc. Clearly, if the atoms are pressed together, the electronic overlap increases, increasing the kinetic energy, which may then have a better chance to overcome the potential energy, localizing the electrons. In 1-D metals, in particular, pressure may increase the overlap between columns, making the material more $3-D$, and thus suppress the Peierls metal to semi-conductor transition at $T_{P}$

In consequence of this, a systematic investigation of the effect of pressure on $T_{P}$ was initiated in Orsay. It was found that in TTF-TCNQ, pressure does not suppress $T_{P}$, but even slightly raises it whilst in TSF-TCNQ, the effect of pressure on raising $T_{P}$ is even more drastic. Effort was continued nevertheless, on HMTSF$T C N Q$, and there it finally succeeded as this material remains metallic down to the lowest temperatures investigated ( 20mK) under pressures in excess of about 2.5 kbar; i.e. the resistivity falls monotonically with temperature, under pressure (Fig. 1(a)).

The discovery of the metallic nature of HMTSF-TCNQ down to the lowest temperatures, opened the way to galvanomagnetic measurements, which were indeed performed immediately. The magnetoresistance was found to be very large; the resistance increases by about a factor of 2 at $3 T$ (Fig. 1(b)). This magnetoresistance is anisotropic, and vanishes for fields parallel to the chains, and changes by about a factor of two when the field is rotated in the plane perpendicular to the chains.

From classical considerations, the magnetoresistance should be appreciable when $\omega_{c} \tau=\mu \mathrm{H} / \mathrm{c}=1$ (where

Fig. 1 (a) - Resistivity of HMTSF-TCNQ as function of temperature at various pressures.

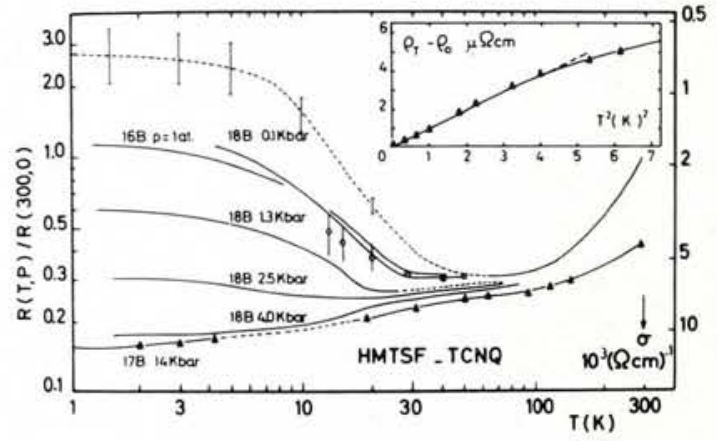




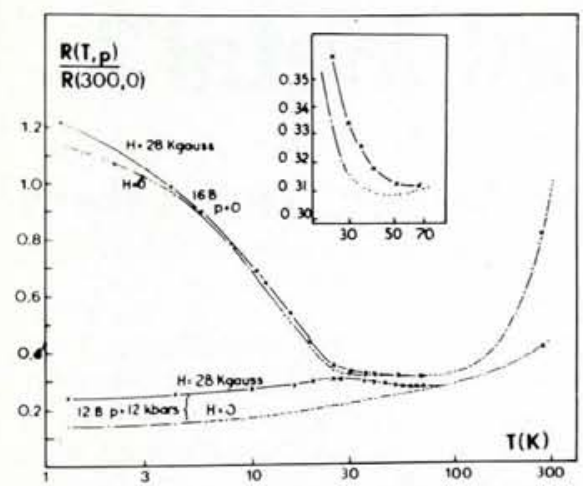

Fig. 1 (b) - Resistivity of HMTSF-TCNQ showing effect of a magnetic field of $2.8 T$.

$\mu=e \tau / m^{*}$ is the mobility) and the quadratic increase in resistivity at low fields indicates a mobility of the order of $10^{4} \mathrm{~cm}^{2} / \mathrm{V} \mathrm{s}$, which is surprisingly high for an organic metal: even ultra-high-quality TTF-TCNQ, at the conductivity maximum at $60 \mathrm{~K}$, does not possess a mobility in excess of $300 \mathrm{~cm}^{2} / \mathrm{V} \mathrm{s}$ or so (and therefore, no appreciable anisotropic magnetoresistance). As the conductivity $\sigma=n e \mu \approx 10^{4}$ $(\Omega \mathrm{cm})^{-1}$ is not very high, this implies that the number of carriers, $n$, must be very small. In these exotic materials, every result must be crosschecked before we can be confident about it. The "standard" way to determine $n$ independently is the Hall effect, as the Hall constant $R_{H}=$ $1 /$ nec yields $n$ directly; moreover, a small $n$ is favourable for this technique, as $R_{H}$ is large and easy to measure. $R_{H}$ as a function of $T$ is shown in Fig. 2. $R_{H}$ increases by three orders from normal to helium temperatures (which is why a very non-linear scale is used in Fig. 2 to display it). Using the standard relations :

$\sigma=n e\left(\mu_{h}+\mu_{e}\right)$;

$R_{H}=(1 /$ nec $)\left(\mu_{h}-\mu_{e}\right) /\left(\mu_{h}+\mu_{e}\right)$; $\Delta \mathrm{e} / \mathrm{e} H^{2}=\mu_{h} \mu_{e} / \mathrm{c}^{2}$,

where $\mu_{h}, \mu_{e}$ are the hole and elec-

Fig. 2 - The Hall constant of HMTSFTCNQ as function of temperature. Due to the 3-order increase at low temperatures, a very non-linear scale is employed.

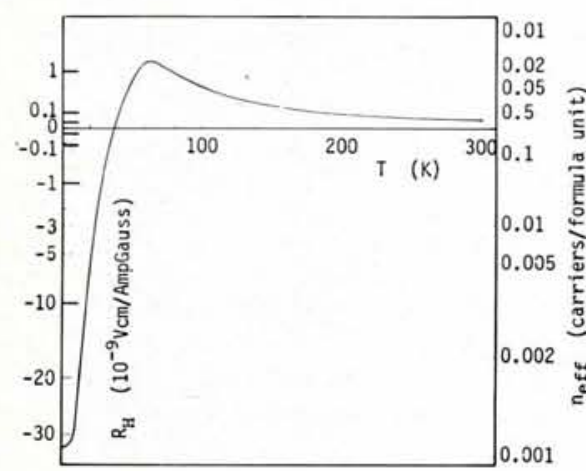

tron mobilities respectively, we get

$n=1.2 \cdot 10^{18}$ carriers $/ \mathrm{cm}^{3}$

$=1 / 2000$ carriers $/$ molecule

$\mu_{h}=12000 \mathrm{~cm}^{2} / \mathrm{V} \mathrm{s}$,

$\mu_{e}=40000 \mathrm{~cm}^{2} / \mathrm{V} \mathrm{s}$ at helium temperatures.

This high mobility, $\mu=e \tau / m^{\star}$, may, in principle, be due to a very long relaxation time $\tau$, or to a small effective mass $m^{*}$. To determine this, we can make use of the magnetic susceptibility. A small effective mass gives rise to a large Landau-Peierls diamagnetism, $\chi_{D}=-\left(e^{2} k_{F}\right) / 12 \pi^{2} c^{2} h^{2} m^{*}$. Such a diamagnetism is indeed observed (Fig. 3), showing that $m^{\star}$ is rather small.

Perpendicular to the chains, the mean free path is considerably smaller; however, at least in the direction perpendicular to the long axis of the molecules, it exceeds (at helium temperatures, under pressure) the separation between the columns; thus the transverse conductivity is coherent (metallic), as well as the longitudinal one.

Thus HMTSF-TCNQ at low temperatures is not a one-dimensional metal, but rather an anisotropic 3-D (or 2-D) semi-metal.

The transition from the 1-D state at high temperatures, to the 3-D state at low temperatures, makes it possible for us to determine experimentally which quantities change and which do not change, in this transition. The longitudinal conductivity does not change significantly (Fig. 1); nor does the anisotropy. These results are at first sight surprising. The reason for this is, that $\sigma=n e \lambda / \mathrm{p}_{F}$; both $n$ and $p_{F}$ decrease, in going from the 1-D to the 3-D state, by three orders, but their ratio is constant, because $n$ is proportional to $p_{F}$ in a 1-D (or nearly 1-D) system. The anisotropy also happens to be essentially the same, $\sigma_{\| /} / \sigma_{\perp} \approx(b / d)^{2}\left(t_{\|} / t_{\perp}\right)^{2}$, where $t_{\|}$and $t_{\perp}$ are the longitudinal and transverse transfer integrals. This formula applies to both 1-D and 3-D states. The quantities that change drastically in going from the 1-D to the 3-D states are, the Hall constant, the magnetoresistance, and the susceptibility. Also, the John Hopkins group found that the thermo-electricpower (TEP) changes from a low, metallic value to a high, semi-metallic one. Several other phenomena associated with this transition will be discussed later.

A metallic conductivity at helium temperatures was also observed recently in $\mathrm{TTT}_{2} \mathrm{I}_{3}$ (tetrathiotetracene triiodide) by Shchegolev's group.

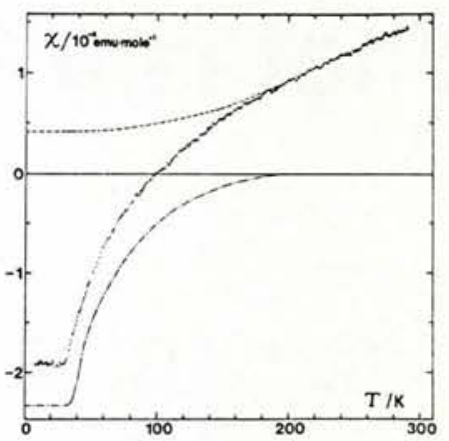

Fig. 3 - The susceptibility of HMTSF$T C N Q$ as function of temperature. The Landau-Peier/s diamagnetism at low temperatures exceeds the Pauli paramagnetism at ambient temperature.

Here, the metallic state exists already at ambient pressure. As in this material only the donor chains conduct, this material should behave rather differently from HMTSF-TCNQ.

\section{The $t_{\perp}-\tau_{\| \prime}$ Diagram}

An illuminating way to illustrate the diffusive to coherent transition of the transverse conductivity is by means of a $t_{\perp}-\tau_{\|}$diagram. Various materials are placed on the diagram according to their values of the interchain hopping integral $t_{\perp}$, and the intra-chain scattering time $\tau_{\|}$(Fig. 4). Variations of temperature, pressure, or other variables, will alter $\tau_{\|}$, and sometimes also $t_{\perp}$, and thus move the material on this diagram. It proves advantageous to express $t_{t}$ and $\hbar / \tau_{\| l}$ in units of $E_{F}$. A $t_{\perp} / E_{F}$ vs. $\hbar / E_{F} \tau$, diagram was first propos$e^{2}{ }^{2}$ in 1976. At that time, $\tau_{\|}$was believed to be due to electron-electron collisions, from the Coulomb interaction $U$; in consequence, a $U-t_{\perp}$ diagram was plotted, and named then "Utopia". However, the diagram, and the conceptual scheme associated with it, do not depend on the origin of $\tau_{\| \prime}{ }^{4}$ ).

Consider two degenerate electronic states, $\varphi_{D}$ and $\varphi_{A}$, on donor and acceptor chains respectively ${ }^{4}$ ). Let an electron be on the donor chain at $t=0$. Then, the wave function as function of time, is given by:

$$
\begin{aligned}
\psi(t) & =\varphi_{D} \cos \left(t_{\perp} t / \hbar\right) \\
& +\varphi_{A} \sin \left(t_{\perp} t / \hbar\right)
\end{aligned}
$$

(assuming degeneracy in energy). After a time $t=\tau_{\|}$a scattering event takes place, destroying the phase relationship between $\varphi_{D}$ and $\varphi_{A}$ The probability of the electron to be found on the acceptor chain, is then given by $\sin ^{2}\left(t_{\perp} \tau_{\|} / \hbar\right)$. If $t_{\perp} \tau_{\|} / \hbar<1$, we can approximate the sine by its 

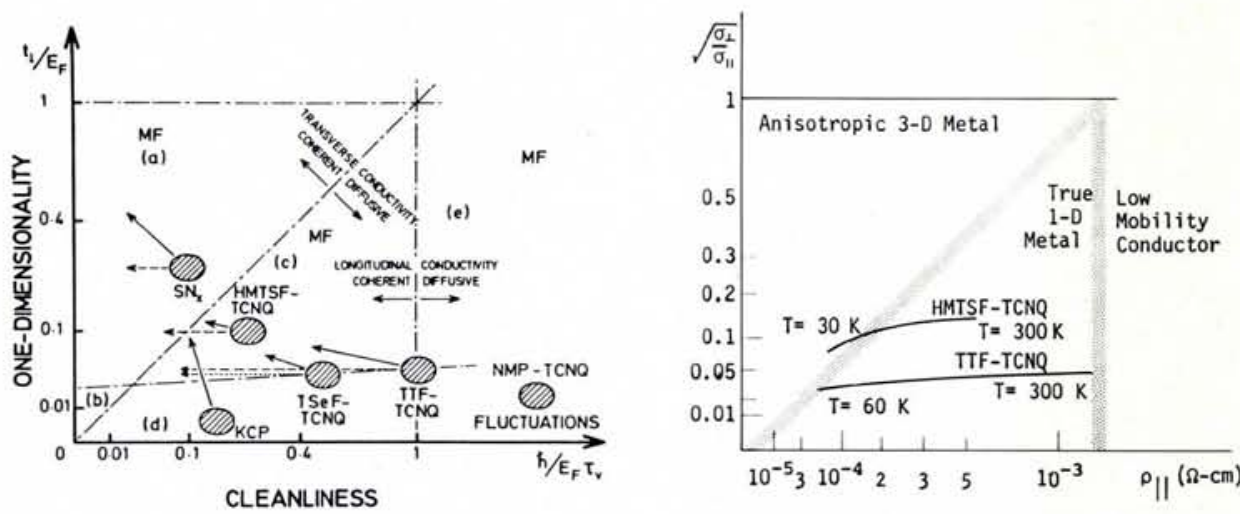

argument, and then the probability for hopping between chains per unit time is given by :

$$
\begin{aligned}
1 / \tau_{\perp} & =\left(1 / \tau_{\| \prime}\right) \sin ^{2}\left(t_{\perp} \tau_{\|} / \hbar\right) \\
& \approx t_{\perp}^{2} \tau_{\|} / \hbar^{2}
\end{aligned}
$$

The electronic motion between chains in this case is diffusive, $\tau_{\perp}$ is an escape time, rather than a collision time, and goes to zero in a perfect crystal $\left(\tau_{\|} \rightarrow \infty\right)$ ). ("Zero" means a time of order $\hbar / t_{\perp}$ ).

When $t_{\perp} / E_{F}<<1$, the system is one-dimensional, while when $t_{\perp} / E_{F}$ $\approx 1$, it is three-dimensional ; thus, the quantity $t_{\perp} / E_{F}$ is a measure of the one-dimensionality of the system. When $\hbar / E_{F} \tau_{\| l}<<1$, the electronic motion along the chain is an uninterrupted, wavelike motion. Thus, in analogy with super-conductors with a long mean free path, this quantity is in a sense a measure of the "cleanliness" of the system, although $\tau_{\text {II }}$ need not be dominated by impurity scattering, and electron-phonon and electron-electron scattering have a similar effect.

With the aid of this diagram (Fig. 4), we can now obtain a much better understanding of the unusual behaiour of the electronic properties of HMTSF-TCNQ at low temperatures.

If $t_{\perp} \tau_{\|} / \hbar>2 \pi$, the electron oscillates back and forth between the chains before being scattered. Actually, in this case, not just a pair of chains, but a whole array has to be considered. The motion between chains is coherent, i.e. wavelike, and we can define a transverse component of the wavevector, $k_{\perp}$ defining a phase-relationship between wavefunctions on different chains. Thus, in the case $t_{\perp} \tau_{\|} / \hbar<1$ (diffusive), the quantum numbers of the electron states are $\left(x, k_{y}, z\right)$ (for motion in the $y$-direction), and in the case $t_{\perp} \tau_{\|} / \hbar$ $>2 \pi$ (coherent), they are $\left(k_{x}, k_{y}, k_{z}\right)$ and we have the ordinary Bloch picture. The region $1<t_{\perp} \tau_{\|} / \hbar<2 \pi$
Fig. 4 (a) - Left the $\tau_{11}-t_{\perp}$ diagram ("Utopia"). A one-dimensional metal is characterized by coherent conduction along the chains, and diffusive conduction perpendicular to them. (b) Right a "poor man's" version of the $\tau_{\| \prime}-t_{\text {, diagram to be employed }}$ when $\tau_{l \prime}-t_{\perp}$ are not sufficiently well known. The parameters were selected for materials like TTF-TCNQ. is a transition region between the diffusive and coherent regions.

Consequently, we draw in the $\tau_{\|}-t_{\perp}$ diagram a line $\hbar / E_{F} \tau_{\| \prime}=t_{\perp} / E_{F}$, (or rather a strip $\left.1 \leq \hbar / \tau_{\|} t_{\perp} \leq 2 \pi\right)$, separating the diffusive and coherent regions. A "one dimensional metal" is thus characterized by $t_{\perp} \hbar / \tau_{\|}<E_{F}$ (If $\hbar / \tau_{\|}>E_{F}$ the Fermi surface is completely washed out, and the motion along the chains is diffusive too, and we have a low-mobility conductor, rather than a metal). When $\hbar / \tau_{\|}<t_{\perp}$, we have an anisotropic yet three-dimensional metal, since the electronic motion is metallic (coherent) in all directions.

In the coherent regime, a gap has opened up in the electronic band structure. We can see this by simply calculating the energy levels, as function of $k_{\mu}$, including $t_{\perp}$ in the hamiltonian. The magnitude of the gap (at $k_{\|}=k_{F}$ ) is given by $2 t_{\perp}$. We call it a "covalency gap" because the states at the bottom are bonding states $\left(\varphi_{D}+\varphi_{A}\right) / V 2$, while those at the top are antibonding ones, $\left(\varphi_{D}-\varphi_{A}\right) / \sqrt{ } 2$, and the lowering in energy of bonding states is known as covalency. The question of whether the material should be insulating, due to the opening of this gap is very delicate.

As the covalency gap opens up, the area of the Fermi surface is diminished, and the electronic density of states falls. This suppresses the Peierls transition temperature $T_{P}$

\section{ETH ${ }_{\text {zwone }}$}

Applications are invitated for occupancy of a chair in

\section{ASTRONOMY}

at the Swiss Federal Institute of Technology and the University of Zurich.

Duties of the new professor will be teaching and research in astronomy, including optical and radio-astronomy and astrophysics.

The successful applicant will have university level teaching experience and proven ability to perform and direct research. $\mathrm{He}$ is expected to cooperate with colleagues in both the Institute of Technology and the University.

Applications should be submitted with curriculum vitae and list of publications before August 31, 1978, to the :

President of the Swiss Federal Institute of Technology,

Prof. H. Ursprung, CH - 8092 Zurich. 


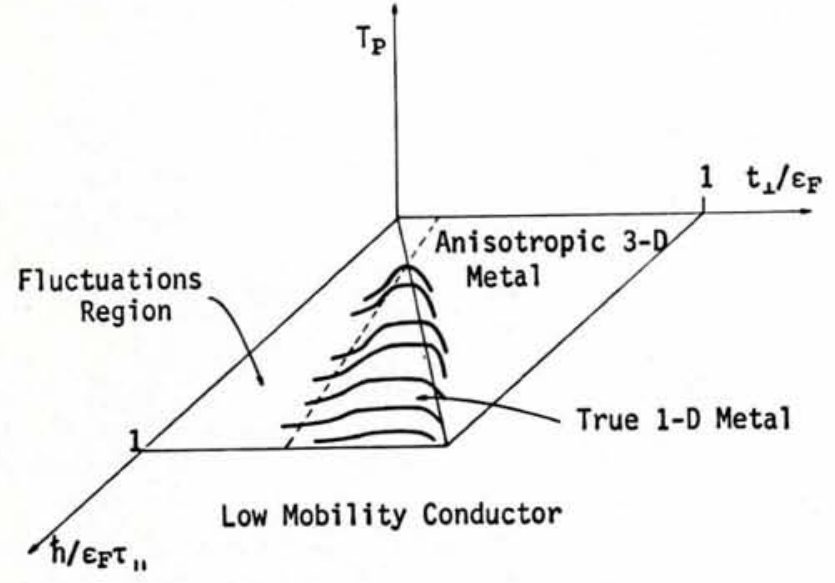

Fig. 5 - Qualitative illustration of the behaviour of the Peier/s transition temperature $T_{P}$ in various regions of the $\tau_{11}-t_{\perp}$ diagram. which accounts for the observation that there is no Peierls transition in HMTSF-TCNQ under pressure, and that the electronic density of states there is small. We show schematically the behavior of $T_{P}$ as function of $\tau_{\|}$, $t_{\perp}$ in Fig. 5.

In the diffusive region, the lifetime for scattering along the chain is so short, that the covalency gap is smeared out. We cannot define a phase-relationship between donor and acceptor states, and therefore there is no covalent bonding between them. In contrast with the situation in chemistry, where covalent bonds are of the order of a few eV, (and therefore the covalent bonds are not broken by thermal agitation at room temperature), here the magnitude of the gap is only a few meV, and thus the covalency can be observed only at low temperatures.

We can define as a measure of the covalency, the average $\left\langle\psi(t), \varphi_{A}\right\rangle$ when $\psi(0)=\varphi_{D}$, i.e. the non-diagonal element $\varrho_{D A}$ of the density matrix. In the diffusive case, it is given by $\left(t_{\perp} \tau_{\| l} / \hbar\right)^{2} / 2=\tau_{1} / 2 \tau$. This quantity is about $10^{-3}$ in TTF-TCNQ at normal temperatures, and becomes greater than one in HMTSF-TCNQ at low temperatures.

The relationship between this quantity and the EPR line width of the conduction electrons has been investigated at IBM and the Bell Telephone Laboratories. In a pure 1-D system, the relaxation process originating from spin-orbit coupling (Elliott Scattering) is suppressed by a selection rule, prohibiting this scattering in one dimension. As the system becomes more $3-D$, this scattering becomes allowed, shortening the lifetime and thus increasing the line width. We see that the line width should be proportional to $\tau_{\| \prime} / \tau_{\perp} \approx \tau_{\| \prime}^{2}$ (in addition to the dependence on the matrix element, which increases strongly with the spin-orbit coupling constant, and thus with the atomic number of the atom on which the scattering takes place ; it can be determined experimentally from the g-shift. Also there is an extra factor $\tau_{\|}^{-1}$ in the Elliott mechanism.) Thus, the line width should increase strongly as the temperature is lowered and also, on going from TTF-TCNQ to TSF-TCNQ, since $\tau_{\|}(D)$ is much longer in the latter compound (as seen, for example, from the thermoelectric power). This is in agreement with experiment, which indicates that the line width is about 10 times larger in TSF-TCNQ than in TTF-TCNQ, when allowance is made for the increased spin-orbit coupling of the seleniums. This is consistent with $\tau_{\|}(D) \approx 1 / 4 \tau_{\| \prime}(\mathrm{A})$ in TTF-TCNQ, ${ }^{4}$ ) vs., $\tau_{\|}(\mathrm{D}) \approx \tau_{\|}$(A) in TSF-TCNQ. $\left(\tau_{\|}(\mathrm{A})\right.$ is comparable in both compounds, because the resistivities are comparable.)

The increase in the EPR line width, as well as the decrease in the escape time $\tau_{1}$ (as measured by NMR), are thus additional indications of the approach to the DCT.

The $\tau_{\|}-t_{\perp}$ diagram is thus an excellent means of classifying the various quasi-one-dimensional metals. Fig. 4 shows several such materials, at ambient temperature and pressure; straight arrows indicate the effect of lowering the temperature, and broken ones - of applying pressure.

In addition to classifying materials, this diagram is also useful in classifying phenomena. There are phenomena associated with the 1-D metallic state, such as the existence of a giant Kohn anomaly, a Peierls transition and the specific behaviour of the electrical resistivity (temperature, pressure, and frequency dependence). There are phenomena associated with the 3-D state, namely the very high mobility, and just recently, Shubnikov- de Haas oscillations were observed in HMTSF-TCNQ in Orsay, proving in a most spectacular way the metallic nature of this state. Also, there are phenomena associated with the DCT, or approach to it; namely, the 3-order increase in the Hall constant, the appearance of an anisotropic magnetoresistance, and of Landau-Peierls diamagnetism; the increase in the EPR line width, and decrease in the inter-chain tunnelling time as measured by NMR.

Another striking phenomenon associated with the DCT is the discovery by Zuppiroli of a tremendous sensitivity of the electrical resistance to radiation damage. In HMTSF-TCNQ at low temperatures, a single radiation-induced defect centre renders a huge region (of the order of a hundred Ångstrom in diameter) insulating. (Defects caused by thermal, or pressure cycling, have similar effects). We can understand this as follows :

Defects cause charge pile-up, by the destruction of the lattice periodicity. This charge induces a potential, which changes the wavevector of the electronic wavefunction; the wavefunctions on neighbouring chains are no longer "in tune", and the coherent movement of electrons between chains (which brings about the $3-D$ semi-metallic state) is impeded. Therefore, the system becomes more one-dimensional, and so more susceptible to a Peierls transition, which renders it insulating.

Another very interesting recent experimental discovery is the observation by Seeger of non-linear I-V curves in TTF-TCNQ, with the conductivity increasing with the electric field. This measurement was carried out at microwave frequencies. One possible interpretation of this experiment is, that carriers are excited across the covalency gap, and in that way reduce it, just like excitation of quasiparticles in superconductors decreases the gap.

This interpretation, hanging "everything" on the covalency gap, is only one of several explanations, at the present stage. In principle, a quasigap due to electron-phonon coupling can perhaps also decrease the effective number of carriers, thus increasing the Hall constant, and create charge density waves which have a tendency to pin, and be unpinned by strong electric fields. We favour the covalency-gap interpretation, at least for the Hall effect, because of the much larger effect in HMTSF-TCNQ, for which $t_{\perp}{ }^{2}$ is about one order of magnitude larger than in TTF-TCNQ. Data on the non-linearity of the I-V curves are 
not yet available for HMTSF-TCNQ (or HMTTF-TCNQ).

\section{Conclusion}

We have shown how the properties of quasi-one-dimensional metals are dominated by the values of the collision time along the chain, $\tau_{\|}$and the interchain tunnelling matrix element, $t_{\perp}$. A true one-dimensional metal is defined by the relations

$t_{\perp}<\hbar / \tau_{\|}<E_{F}$
As $\hbar / \tau_{\|} t_{\perp}$ diminishes, a transition to an anisotropic, three-dimensional, high mobility semi-metal takes place. This Diffusive to Coherent Transition (alias, the opening of a covalency gap) does not have a significant effect on the longitudinal and transverse conductivities, but greatly affects the Hall constant, magnetoresistance, diamagnetic susceptibility, EPR line width, NMR inter-chain tunnelling time, the Peierls transition temperature $T_{p}$ and its pressure dependence, and the sensitivity of the resistivity to radiation damage.

Thus, we have a unified phenomenological description of a very wide variety of phenomena, which does not depend upon the microscopic origin of the collision time $\tau_{\|}$.

\section{References}

1. "Low Dimensional Co-operative Phenomena", Editor H.J. Keller, NATO AS1 series B7, (Plenum Press, New York) 1975 2. "Chemistry and Physics of One-Dimensional Metals", Editor H.J. Keller, NATO AS1 series B25, (Plenum Press, New York) 1977

3. "Organic Conductors and Semiconductors", Conference Proceedings, Siofok, Hungary, 1976, Lecture Notes in Physics, 65. (Springer Verlag) 1977

4. SODA, G., JEROME, D., WEGER, M., ALIZON, J., GALLICE, J., ROBERT, H., FABRE, J.M. and GIRAL, L. J. Physique 38 (1977) 931

5. PIPPARD, A.B. Reports on the Progress of Physics 23 (1960) 176

\section{Upsilon Particle Produced at Desy}

The upsilon-particle, the first signs of which were seen in proton-nucleus collisions, has now been produced for the first time in electron-positron collisions, where it can be studied with more precision. The rate at which the new particle is produced and its long life-time indicate that it is very probably a compound, made up of two quarks of a new type. Although the quarks are new, the experiments support the general quark picture surprisingly well.

The experiments were performed at the $\mathrm{e}^{+} \mathrm{e}^{-}$storage-ring DORIS, modified substantially to reach the energy of the upsilon particle. Orinally, DORIS had been designed for a total centre of mass energy of 7 $\mathrm{GeV}$, obtained in the collision of two beams of $3.5 \mathrm{GeV}$ each, but the 48 bending magnets had fortunately been built powerful enough to deal with beams of up to about $4.5 \mathrm{GeV}$ and to obtain the necessary RF power, cavities from the storage ring PETRA (now under construction) were substituted for the normal cavities. For the upsilon search, the total energy of DORIS was upgraded to $9.5 \mathrm{GeV}$.

The measurements of the upsilon were carried out with the doublearm-spectrometer DASP and the detector PLUTO incorporating a superconducting magnet. This has been improved substantially over the past year to cover more than $96 \%$ of the solid angle by a system of shower counters, which now permit the detection of photons in addition to charged particles.

The first result from the DESY teams * was the exact determination of the mass of the upsilon which came out to be $(9.46 \pm .01) \mathrm{GeV} / \mathrm{c}^{2}$; the width of the mass-distribution was too small to be measured, indicating therefore a long life-time. This fact agrees with the hypothesis that the upsilon is a relatively stable compound state of two new quarks, each assumed to have a mass around $5 \mathrm{GeV} / \mathrm{c}^{2}$. According to current theories, the DESY data show that the new quarks have an electric charge of $1 / 3 \mathrm{e}$. The characteristic property of these quarks is a new quantum-number called (arbitrarily) "beauty" (to add to the existing quantum numbers: "up", "down", "strange", and "charm ").

During the scan for the upsilon another very important discovery was made: particle production at these energies proceeds mainly via an intermediate state which is believed to be a high energy quark-antiquark pair. The two quarks immediately transform into stable particles which are then detected as two characteristic "jets". This fact is another important piece of evidence in favour of the hypotheses of the quark structure of matter. In addition, these jet events will provide important information to check the quark theory in detail and to learn about the forces acting between quarks.

\footnotetext{
* The two measuring teams included physicists from DESY, the Universities of Dortmund, Hamburg, Heidelberg and Lund, the Physics Institute of the RWTH at Aachen, the Gesamthochschules of Siegen and of Wüppertal, and included also physicists on detachment from the Universities of Tel Aviv, Humboldt and Purdue.
}

\section{Albert Einstein Centenary}

\section{IN HONOUR OF THE ONE HUNDRETH ANNIVERSARY OF THE BIRTH OF ALBERT EINSTEIN}

SPONSORED BY: UNESCO, IUPAP, INT. COM. ON GRG, CERN, SWISS ACAD. OF SCIENCES, UNIVERSITY OF BERN, THE SWISS AUTHORITIES, THE ALBERT EINSTEIN FOUNDATION

\section{A PUBLIC CONFERENCE 13-17 MARCH, 1979 \\ in the Alfa Conference Centre BERN}

with the participation of the world's leading theoretical physicists.

No registration fee will be charged.

BUT prior registration is necessary. Please use form opposite.
Complete and return the form below by 30 Sept., to: Dr. Alan Held, Dept. of Theor. Phys., Sidlerstrasse 5, CH-3012 BERN.

Name :

First Name :

Address :

\begin{tabular}{|c|c|}
\hline Tel. : & Telex: \\
\hline Accom & \\
\hline Date : & Sig. : \\
\hline
\end{tabular}

